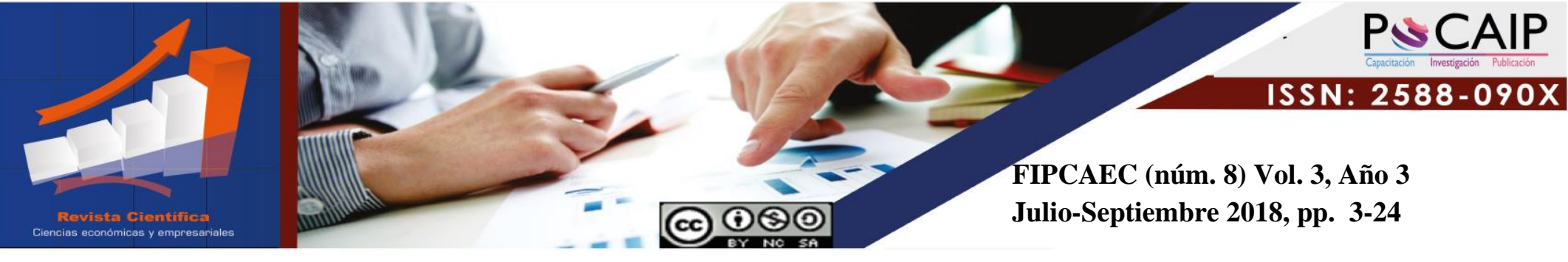

DOI: $10.23857 /$ fipcaec.v3i8.55

\title{
Las Pymes y su problemática empresarial
}

\section{SMEs and their business problems}

\section{PME e seus problemas de negócios}

\author{
Katty Gisella Zambrano-Alcívar ${ }^{1}$ \\ kattygiza@hotmail.com \\ https://orcid.org/0000-0003-1886-6147
}

Correspondencia: kattygiza@hotmail.com

* Recepción: 28/ 04/ 2018 * Aceptación: 16/06/ 2018 *Publicación: 15 /07/ 2018

Doctora en Ciencias Administrativas, Magíster en Educación y Desarrollo Social, Diploma Superior en Liderazgo Educativo, Magíster en Educación y Desarrollo Social, Magíster en Gerencia de Proyectos Educativos y Sociales, Licenciada en Ciencias de la Comunicación Especialización Periodismo, Tecnóloga Pedagógica en Informática, Técnico Superior Especialidad Programación de Sistemas, Profesora de Segunda Enseñanza Especialización: Informática, Docente de la Universidad Laica Eloy Alfaro de Manabí, Extensión Chone, Chone, Ecuador. 


\title{
Resumen
}

Actualmente las empresas desempeñan un papel importante dentro del entorno, satisfaciendo las necesidades fisiológicas, psicológicas y de autorrealización social, como los modelos de desarrollo tecnológico y económico de los países. Se realizó una revisión bibliográfica relacionada con las micro, pequeñas y medianas empresas, manifestando algunas de sus peculiaridades: en entorno mundial, definición, clasificación, desarrollo en América Latina, dimensión empresarial y desarrollo económico. Se concluye, son importante no solo por su participación en la creación del PIB, sino también por ser una fuente generadora de empleo extensiva.

Palabras claves: Empresa; pequeñas y medianas empresas (Pymes) globalización; PBI.

\begin{abstract}
Nowadays companies play an important role within the environment, satisfying the physiological, psychological and social self-realization needs, such as the models of technological and economic development of the countries. A bibliographic review related to micro, small and medium enterprises was carried out, showing some of its peculiarities: in a global environment, definition, classification, development in Latin America, business dimension and economic development. It is concluded, they are important not only for their participation in the creation of GDP, but also for being a source of extensive employment.
\end{abstract}

Keywords: Company; small and medium enterprises (SMEs) globalization; GDP.

\section{Resumo}

Atualmente, as empresas desempenham um papel importante no meio ambiente, satisfazendo as necessidades de auto-realização fisiológica, psicológica e social, como os modelos de desenvolvimento tecnológico e econômico dos países. Foi realizada uma revisão bibliográfica relacionada às micro, pequenas e médias empresas, mostrando algumas de suas peculiaridades: ambiente global, definição, classificação, desenvolvimento na América Latina, dimensão empresarial e desenvolvimento econômico. Conclui-se que são importantes não apenas por sua participação na criação do PIB, mas também por serem uma fonte de emprego extensivo. 
Palavras-chave: Empresa; globalização das pequenas e médias empresas (PME); PIB.

\section{Introducción}

El objeto central de estudio de la teoría administrativa lo constituyen la pequeña y mediana empresa, las investigaciones se han centrado fundamentalmente en el análisis de la perspectiva económica y en el ámbito de la gestión empresarial. (Zapata Guerrero, Edgar Enrique 2004).

Los sistemas económicos de distintos países en los últimos años, han declarado interés hacia una categoría específica dentro de las empresas, concerniente a las pequeñas y medianas empresas, razonando que contribuyen de manera sustancial al desarrollo económico, generando empleo e ingreso, avivando el interés de empresarios, profesores de gerencia, organismos multilaterales y políticos; estas empresas son fáciles de establecer, pueden instituir el inicio de grandes empresas y, si bien pueden producir bienes tan tradicionales como la artesanía, la repostería, entre otros, o servicios tan sencillos como, el de taxis, de comida rápida dentro de algunos otros, también han penetrado con éxito al mundo tecnológicamente avanzado como el de la informática. Se está a la mira cada vez más a los esfuerzos orientados a adecuar las organizaciones al complejo escenario en que se mueven; cambios de reglas de juego, incremento de la competencia, apertura al mundo a través de la tecnología, hacen al cliente mucho más exigente, modificando sus demandas y necesidades. La Gestión basada en los Procesos, surge como un enfoque que centra la atención sobre las actividades de la organización, para optimizarlas. (Rojas, M., Briceño, M. 2007).

Es de importancia abordar brevemente el surgimiento de las Pymes; Solo a partir de la década de los 90` los economistas y estudiosos de las organizaciones le toman interés y atención a las pequeñas y medianas empresas, se encontraban focalizados hacia el tratado de las Grandes Empresas, otorgándole a las mismas una unidad conceptual propia y singular, con características y dinámicas particulares diferenciadas de las Grandes Empresas; esto fue debido al enfoque dominante en materia empresarial que imperó durante las décadas previas, conocido como la era de la producción en serie, el cual se alineaba al modelo de industrialización promovido por Henry Ford a inicios del siglo XX. Las empresas Grandes eran valoradas como el templo de la formalidad ya que operaban reguladas por leyes laborales y se beneficiaban con 
incentivos gubernamentales. Por el contrario las empresas pequeñas y medianas eran consideradas como pertenecientes a sectores informales de característica familiar. (Madrid C. 2018).

En la década del 70' se produce la conocida crisis productiva mundial que puso en jaque al paradigma Fordista, debido a que la envergadura y rigidez de sus estructuras eran incapaces de adaptarse a los nuevos mercados de cambio continuo. A partir de ese momento es que se las empieza a valorar como agentes de cambio, con destacable capacidad de adaptación e innovación de productos y servicios, con bajo capital, costos y riesgos involucrados a las Pymes, su virtuosa proliferación ha nutrido las economías de los distintos países que componen el globo. (Madrid C. 2018).

Literaturas estudiadas las Pymes se conceptualiza como empresa "...la unidad económico social en el que el capital, el trabajo y la dirección se coordinan para lograr una producción que responda a los requerimiento del medio humano en el que la propia empresa actúa". Se compendia como una unidad que busca satisfacer la demanda del mercado al cual pertenece mediante la producción de bienes o servicios, debiendo para ello coordinar efectivamente sus recursos técnicos, humanos y financieros. (Madrid C. 2018).

\section{Clasificación de las Pymes}

Existe una gran variedad en el mundo de formas de discurrir y definir a las micro, pequeñas y medianas empresas, dependiendo de las necesidades propias de cada país o de los objetivos que se persigan. Cada país, tomando en cuenta su características propias, sus necesidades singulares y los intereses generados de por medio, se pueden esgrimir los argumentos necesarios para aplicar determinada clasificación y con base en ella aplicar las políticas, medidas y estrategias económicas tendientes a propiciar el desarrollo de dichos estratos empresariales. (Saavedra G., M L y Hernández C., Y. 2008).

Refieren importantes investigadores que cada país define a las Pymes utilizando pautas esencialmente cuantitativas que si bien tiene ciertas características similares, también tiene diferencias significativas. Incluso en algunos casos, no hay consenso dentro del mismo país. En la mayoría de los casos, las variables consideradas son cantidad de personal, cifra de ventas y 


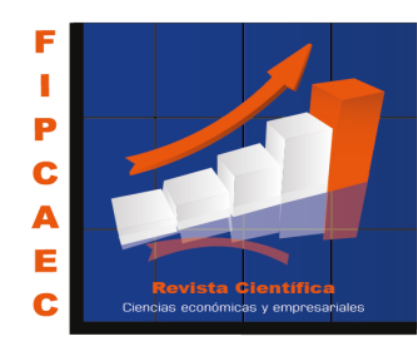

niveles de inversión en activos. Los organismos internacionales con el fin de canalizar los apoyos financieros y logísticos, también se han dado a la tarea de establecer clasificaciones para este sector, ejemplificamos: de acuerdo la OCDE, las empresas se pueden clasificar en: (OECD 2005) Según el número de trabajadores:

- Muy pequeñas : 0-9 trabajadores

- Pequeña : 20-99 trabajadores

- Medianas : 100- 499 trabajadores

- Grandes $\quad$ : $500 \mathrm{y}+(\mathrm{OECD}(2005)$.

De la misma manera, la comisión de las Comunidades Europeas en el Diario Oficial, 2003, exhibe la recomendación 2003/361/CE, del 6 de mayo que contempla la siguiente clasificación: (Saavedra G., M L y Hernández C., Y. 2008)

\begin{tabular}{llll} 
& Empleados & Ventas & Activo \\
\hline Micro empresas & $\geq 9$ & Hasta 10 millones & Hasta 10 millones \\
Pequeña & $\geq 49$ & Hasta 10 millones & Hasta 10 millones \\
& & & \\
Mediana & $\geq 249$ & Hasta 50 millones & Hasta 43 millones \\
\hline
\end{tabular}

Según se ha visto las clasificaciones pueden ser determinadas por entes organizados en bloques económicos como es el caso de la OCDE, la CE o la CEPAL, así también se encuentran clasificaciones que realizan los institutos de estadísticas de cada país como es el caso del INSEE de Francia. De otro lado, están las clasificaciones que utilizan los diversos organismos que agrupan a empresarios como es el caso de las SBA de Estados Unidos de Norteamérica. (Saavedra G., M L y Hernández C., Y. 2008) 
En América Latina no existe homogeneidad al definir a las Pymes lo cual dificulta su estudio y análisis. Es necesario señalar que muchos de los países presentan más de un criterio para definir a las Pymes, que varían dependiendo del sector en que se encuentran. (Saavedra G., M L, Hernández C., Y 2008).

\section{Ejemplificamos:}

En Argentina donde el criterio de clasificación son las ventas anuales, la definición difiere (bajo el mismo criterio) dependiendo de si la empresa es manufacturera, comercial o de servicios. (Saavedra G., M L, Hernández C., Y 2008).

En Brasil existen diversas definiciones de micro, pequeña y mediana empresa

Colombia se maneja los parámetros de empleo y activos y, según la Ley para el Fomento de la Micro, Pequeña y Mediana Empresa, Ley 590, las MIPYMES. Para la clasificación de aquellos micros, pequeñas y medianas empresas que presentan combinaciones de parámetros, el factor determinante es el de activos totales. (Saavedra G., M L, Hernández C., Y 2008). Así mismo en Brasil existen diversas definiciones de micro, pequeña y mediana empresa. (Saavedra G., M L, Hernández C., Y 2008)

Costa Rica, maneja criterios de empleo, ventas, activos, inversión en maquinaria, equipo y herramientas. . (Saavedra G., M L, Hernández C., Y 2008)

En Venezuela, no existe una definición (ni conteo) oficial para las empresas comerciales y de servicios; sólo de las manufactureras. A partir de la promulgación del Decreto Ley para la Promoción y Desarrollo de la Pequeña y Mediana Industria - PYMI (12/11/2001), se introduce una nueva definición que establece como parámetros para la PYMI, el número de empleados y el nivel de ventas en unidades tributarias. (Saavedra G., M L, Hernández C., Y 2008)

Con referencia a lo anterior la mayoría de los casos la categorización de las empresas, se hace principalmente sobre la base de la cantidad de personal empleado, vinculado con otros factores cuantitativos como volumen anual de facturación, valor de activos productivos y otros, ya sea tomados como límites absolutos, o mediante la combinación de uno o más de ellos. Cada país 
utiliza un criterio de clasificación de acuerdo con las necesidades sociales, políticas y económicas que le rodean. (Saavedra G., M L, Hernández C., Y 2008).

La producción de las Pymes está orientada esencialmente hacia el mercado interno y su desempeño está fuertemente influenciado por las condiciones macroeconómicas (Pérez y Stumpo, 2002). Por lo tanto, situaciones de inestabilidad macroeconómica y caída del PIB afectan fuertemente tanto el nivel de producción como el empleo de estas empresas.

\section{Pymes en América Latina}

El tópico de las Pyme en América Latina se torna cada vez más interesante y específicamente en estos momentos de globalización. No obstante, no se encuentra simplemente disponible la información mínima que conceda conocer la situación de estas empresas en cuanto a su definición, al número de estas empresas, la conformación sectorial y su importancia económica. (Saavedra G., M L y Hernández C., Y. 2008),

Refieren importantes investigadores que el aparecimiento en América Latina de las Pymes, se engendran primordialmente por dos caminos.

- Empresas propiamente dichas, con una adecuada estructura, que les permite una adecuada gestión empresarial: este tipo de organización es capital- intensivas y se desarrolla dentro del sector formal de la economía. Donde ubican fundamentalmente las pequeñas y medianas empresas.

- Empresas familiares: caracterizadas por una gestión, orientada a la supervivencia sin prestar demasiada atención a temas tales como el costo de oportunidad del capital, o la inversión que permite el crecimiento. En este contexto, se ubican las microempresas que surgen asociadas al fenómeno del autoempleo como forma de subsistencia. (Valdés Díaz de Villegas, JA, Sánchez Soto, GA 2012).

En Latinoamérica la gran mayoría de las Pymes son organizaciones informales, brotadas de los proceso de atomización social producto de la integración de estas economías al proceso de globalización, con el uso de un paradigma de política económica neoliberal monetarista, sin que se hayan operado cambios profundos en las estructuras competitivas de la actividad empresarial, 
ni en el marco jurídico institucional que permite el funcionamiento de la actividad económica. (Valdés Díaz de Villegas, J A, Sánchez Soto, G A 2012).

Actualmente el crecimiento de la región está afectado en el ámbito geopolítico, sin embargo, conciben nuevos espacios que pueden ser aprovechados; el lento crecimiento económico, el menor dinamismo del comercio internacional, la aceleración de la revolución digital que está modificando los patrones de consumo y de producción en todo el mundo y las nuevas demandas globales vinculadas a los Objetivos de Desarrollo Sostenible y la Agenda 2030 para el Desarrollo Sostenible; pudiéndose afirmar que estos acontecimientos representan desafíos y oportunidades para que los países de América Latina puedan encarar un nuevo estilo de desarrollo más justo y sostenible. (Dini M, Stumpo G. 2018).

Es visionado y notorio que las micro, pequeñas y medianas empresas (Pymes) son un componente fundamental del tejido empresarial en América Latina. Esta categoría se manifiesta en varias dimensiones, como su participación en el número total de empresas o la creación de empleo. Ello se contrapone a una participación al producto interno bruto (PIB) regional de tan solo el 25\%, situación que contrasta con la de los países de la Unión Europea (UE) donde las Pymes alcanzan, en promedio, el 56\%. (Dini M, Stumpo G. 2018).

Es de referencia que las micro, pequeñas y medianas empresas (Pymes) se caracteriza en América Latina por una estructura productiva heterogénea y la especialización en productos de bajo valor agregado influyen de manera decisiva sobre el desempeño de estas empresas y se manifiesta en la productividad y en una muy baja participación en las exportaciones. Estos rezagos en productividad y capacidad exportadora representan uno de los aspectos de la elevada heterogeneidad estructural de las economías de la región. (Dini M, Stumpo G. 2018).

Uno de los aspectos indicado con mayor periodicidad para justificar las políticas de protección en favor de las Pymes es el en el empleo formal, el cual es relevante

Las Pymes latinoamericanas tienen una amplia presencia en todos los sectores productivos desde el comercio y la industria hasta los servicios, la salud o los sistemas financieros, y una importante influencia en la creación de tejido social. De hecho, se calcula que cerca del $60 \%$ de 
latinoamericanos trabajan en empresas de cinco o menos empleados. (América Latina: en busca de Pymes más competitivas. 2016)

\section{Importancia de las Pymes en América Latina}

Las Pymes en Latinoamérica juegan un importante papel de carácter social, tanto por su papel como creadoras de empleo y amortiguadores del problema de desempleo al ser intensivas en este factor, como por ser un instrumento de cohesión y estabilidad social al brindar oportunidades de empleo a colectivos semi o escasamente cualificados. (Saavedra G., M L y Hernández C., Y. 2008).

- Empleo: entre el 25\% y el $45 \%$ de los ocupados

- Producción: entre el 15\% y el 25\%

- Exportaciones: entre 1\% y el 3\% (Vergara M S .2005)

La distribución espacial de las empresas de la región obedece a los patrones de desarrollo centralizados de América Latina. La mayor parte de las empresas de la región se ubica en las capitales y ciudades más importantes. Aunque esto no es diferente en los países desarrollados, en nuestros países el grado de concentración es bastante mayor. (Saavedra G., M L y Hernández C., Y. 2008).

Se debe de destacar que en busca del sustento para afrontar las responsabilidades del diario vivir en América Latina se ha desarrollado una economía informal significativa, ante la aparición de pequeños negocios de subsistencia, situación que ha frenado el emprendimiento como proceso necesario de autoempleo al limitar el avance tecnológico, además de afectar seriamente a muchos negocios que operan dentro de la formalidad. (Valdés Díaz de Villegas, J A, Sánchez Soto, G A 2012).

\section{Perspectiva regional en el desarrollo de las Pymes}

La dimensión de los desafíos contenidos en los procesos de transformación de los sistemas productivos de la región plantea más de un tema que debería ser abordado en una perspectiva regional, mediante una acción coordinada de los actores del fomento productivo de la región. 
Citamos (...) Acciones para el desarrollo de las Pymes planteadas por la comisión Económica de América Latina (CEPAL): Alistamos.

- Las autoridades rectoras de las políticas orientadas a las Pymes de los países de la región podrían elaborar principios generales que guíen el diseño e implementación de las políticas de fomento dirigidas a las Pymes, generando un documento de referencia, en el estilo del Small Business Act de la Unión Europea.

- Cimentar una instancia de encuentro de los representantes de las instituciones rectoras de las políticas y de las entidades privadas y académicas que participan en su elaboración o implementación, que periódicamente razone los avances de las políticas, comparta las experiencias y los aprendizajes y formule sugerencias a los gobiernos

- Dotar esta instancia de encuentro de los instrumentos, capacidades y recursos necesarios para realizar investigaciones, diseñar propuestas y realizar acciones de apoyo a los distintos gobiernos en las distintas áreas del fomento de las mipymes

- Establecer una plataforma virtual que facilite el registro y el intercambio de las experiencias implementadas en diseño, gestión y evaluación de políticas para mipymes y que promueva una gestión activa del conocimiento a través de encuentros periódicos, sobre aspectos temáticos determinados.

- Concretar una estrategia para la construcción de sistemas de información basados en registros administrativos que permitan generar y mantener bases de datos actualizadas y detalladas sobre las mipymes y su desempeño.

- Ejecutar anualmente un plan de estudios y actividades de asistencia técnica para los gobiernos de la región, a fin de ampliar y profundizar los análisis de buenas prácticas, la formulación de recomendaciones y la realización de actividades de formación en temas de interés, como compras públicas para el desarrollo de las Pymes, incorporación de nuevas tecnologías digitales en las Pymes, desarrollo de cadenas productivas en sectores dinámicos, construcción de mecanismos institucionales para la dinamización de los sistemas productivos locales, mecanismos de financiamiento para cadenas productivas, redes empresariales o esquemas asociativos de empresas, entre otros. (Dini M, Stumpo G .2018) 


\section{Panorámica de las Pymes en algunos países de América Latina}

\section{Ecuador}

Ecuador posee un apremiado crecimiento y desarrollo en lo referente a las Pymes; no obstante estas deben enfrentar la competencia de grandes empresarios con poder económico que provienen de ciudades grandes, quienes se diferencian por su alto nivel de productividad el cual es alcanzado por la correcta aplicación de la administración de operaciones en sus proceso, aspecto que las pone en desventaja. Las Pymes son fuente generadora de empleo, es primordial que las mismas logren alcanzar un desarrollo sostenible en el tiempo a través de procesos y productos de calidad. (Yance Carvajal C, Solís Granda L, Burgos Villamar I, Hermida Hermida L. 2017).

En este orden de ideas se puede expresar que las pequeñas y medianas empresas en Ecuador constituyen el 75,0 \% de las compañías en el país y concentran más del 70,00\% de los empleos (Estupiñan, 2015). Los triunfos que se producen cada día son ejemplo verdadero de su influencia e importancia. Las Pymes sobre todo las familiares enfrentan dos desafíos permanentes que pueden llegar a constituir fuertes amenazas a su sobrevivencia: el aprendizaje y la práctica de nuevas formas de administración, así como la reinvención constante del negocio, debido a factores internos y externos que intervienen en el mercado, y que para transformase de una empresa doméstica, a una gran empresa, es necesario realizar cambios fundamentales en la forma de cómo se administra, entrar en un proceso de integración y automatización de la información, estandarización de los flujos de procesos, para volverse cien por ciento eficientes. (Cervantes Molina, Ximena P. Oviedo Bayas, B. 2018).

El INEC, (2016), reseña que alrededor de 179.830 pymes se encuentran en el país donde la provincia del Guayas abarca con un total del 32.67\% que representa a un total de 58.574 empresas de este tipo, seguido de la provincia de Pichincha con un aporte del $27.95 \%$ equivalente a una cantidad de 50.269 pymes y por último la provincia de Manabí con un $4.69 \%$ que equivale a 8.438 empresas. \%. El 89\% de las empresas del Ecuador son familiares. 
Las principales actividades a los que se dedican las empresas en el país son tres: industria, comercio y servicios en donde la industria es la que tiene gran cantidad de empresas presentes en el territorio nacional. (Delgado Delgado DD., Chávez Granizo GP. 2018)

\section{Tipos de Pymes en el Ecuador}

De acuerdo a la información publicada por La Ley de Compañías en el Ecuador entidad que regula a las empresas en general aún no existe un órgano regulador de las Pymes., razón por la cual muchas Pymes, no se encuentran debidamente reguladas, debido a que muchas operan de manera informal, por lo general la conforman personas naturales, sin embargo deben regirse de acuerdo como establece la legislación ecuatoriana para estos tipos de empresas y negocios en el país. (Yance Carvajal C, Solís Granda L, Burgos Villamar I, Hermida Hermida L. 2017).

Resulta oportuna expresar que las Pymes no está solamente en capacidad de generar fuentes de trabajo, también generan competencia al introducirse al proceso productivo en condiciones adecuadas, debido al progreso de una gran variedad de iniciativas efectuadas por una diversidad de actores. Acorde va creciendo la microempresa, también van ampliándose la demanda y los servicios de ayuda por parte del estado y las instituciones públicas y privadas. Es importante mencionar a nivel nacional el crecimiento que han logrado en la última década a partir de la dolarización, donde los empresarios encontraron mayor estabilidad sobre el campo monetario e inflacionario. (Yance Carvajal C, Solís Granda L, Burgos Villamar I, Hermida Hermida L. 2017).

\section{Impedimentos de las Pymes}

La subsistencia de las Pymes ecuatorianas subsisten se debe a un mercado cambiante que a diario abre nuevos campos para satisfacer nuevas necesidades. No obstante, al no responder a estas oportunidades por la falta de recursos financieros, no pueden perdurar en el mercado. Las empresas pequeñas tienen una mayor dificultad para acceder a financiamiento debido a que tienen un alto riesgo y plazos adecuados por parte del sector privado. Por estas razones es que usualmente las Pymes se ven obligadas a recurrir a pagar mayores intereses y a recibir capital riesgoso. 
Resumiendo: Los obstáculos son factores que no permiten el desempeño normal y eficaz de las Pymes, con ello las ideas de su producto o servicio se ve estancada debido a muchos factores entre ellos internos como la situación económica del país, el acceso a financiamiento y el sistema tributario que juntos ocasionan dificultades a estas empresas. (Delgado Delgado D D , Chávez Granizo G P . 2018)

Situación actual.

- No existe crédito de desarrollo (capital de riesgo).

- Potencial de crecimiento de Pymes limitado por falta de acceso real a crédito,

- 2 de cada 6 emprendimientos reciben crédito

- Tasa de interés más alta limita acceso a crédito

- Limitado uso de crédito público

- Limitada capacitación en Pymes para desarrollar modelo de negocios

Investigación realizada en el Cantón Empalme relacionada con las Pymes manufactureras, arrojo que existen 1495 establecimientos económicos de los cuales 140 corresponden a mi pymes manufactureras mismas que se encuentran en diferentes actividades económicas como calzado, confecciones, elaboración de ladrillos, ebanisterías; entre otros; generando en su cadena de valor una serie de procesos mismos que son llevados por parte de los dueños de los establecimientos de una manera empírica. Se pudo evidenciar que sus propietarios desconocen de técnicas de gestión por procesos es así que el 73,17\% de los entrevistados no lleva planes de mejora a fin que le permitan mejorar sus acciones; el 67,07\% carece de conocimientos en cuanto a las consecuencias de las fallas en el proceso; el 97,56\% no posee ningún sistema para detectar fallas y el nivel de actualización de procedimientos para ejecutar las operaciones es bajo con un 73,17 por ciento.( Cervantes Molina, Ximena Paola, Oviedo Bayas, Byron. 2018).

\section{Colombia}

La economía de Colombia, se ha visto sacudida por fenómenos sociales tales como el narcotráfico, la violencia y los altos niveles de pobreza, no es una excepción, haciéndose 
prioritario el desarrollo empresarial y el sostenimiento de las Pymes como un paso adelante en su desarrollo económico.

El ministerio de Comercio, asegura que "las Pymes son parte fundamental de la economía en la Colombia moderna que se asevera, por el peso que tienen en la generación de empleo y por lo que representan en términos del PIB1, en tal ocasión se fortalece su competitividad con la Política de Desarrollo Productivo lo que le permitirá crecer por medio de la internacionalización". Sin embargo, la informalidad que manejan las Pymes, impiden ese objetivo de internacionalización. Según el DANE, las Pymes generan alrededor de 67. 0\% del empleo y aportan 28\% del Producto Interno Bruto (PIB), lo cual es una clara muestra del aporte que realizan las Pymes a la economía del país. (Quintero Reatiga J. 2018).

El país cuenta con leyes y planes para incentivar el empoderamiento de las Pymes; sin embargo los esfuerzos del estado para fomentar la creación de empresa y consolidación se han quedado cortos, se deben buscar medidas para garantizar el desarrollo de las Pymes y disminuir considerablemente la tasa de mortalidad. (Quintero Reatiga J .2018)

\section{México}

A pesar de que el mayor número de las Pymes en México, operan en la informalidad, constituyen una parte determinante de la estructura económica del país, por su alto impacto en la generación de empleos y en la producción nacional. Por tanto aunque pueda considerarse contraproducente su informalidad, instituyen un factor generador de ingresos, que tiene incidencia, fundaméntales, en la demanda de consumo, jugando su papel como autoempleo de subsistencia, una característica que es un denominador común en el resto de América Latina. (Valdés Díaz de Villegas, J A, \& Sánchez Soto, G A 2012).

Cabe agregar que las estadísticas del INEGI, muestran la existencia de un proceso piramidal de la estructura empresarial en México, el 95.2\% son microempresas, que ocupan al $45.6 \%$ de todo el personal, en tanto que las pequeñas empresas son $4.3 \%$ y emplean al $23.8 \%$; por último las medianas empresas son el $0.3 \%$ y ocupan al $9.1 \%$ del personal disponible, y el restante $0.2 \%$ son empresas de gran tamaño que emplean al $21.5 \%$ de las personas reportadas. (Instituto Nacional de Estadística y Geografía 2009), (Valdés Díaz de Villegas, J A, \& Sánchez Soto, G A 2012). 
En la economía mexicana una de las características fundamentales es su colocación a la exportación por la estrechez de su mercado doméstico; sin embargo los datos muestran que no existe una correspondencia entre el modelo exportador y la participación de las Pymes en la actividad exportadora del país.

\section{Dimensión empresarial y desarrollo económico de las Pymes}

Se rivaliza en la nueva coyuntura, la repercusión de los cambios en la escena económica mundial sobre la competitividad de las Pymes; de todo esto se desprende el papel esencial que ocupa el sector de las Pymes en las economías actuales con independencia de su nivel de desarrollo, ya se considere su peso sobre el número total de empresas o su aportación al PIB o al empleo. En gran número de los países de la OCDE, las Pymes representan entre $96 \%$ y 99\% del número total de empresas y aportan aproximadamente una sexta parte de la producción industrial, entre 60 y $70 \%$ del empleo en ese ámbito, así como la mayoría de los empleos en el sector servicios. En países como Portugal, Italia, España, Bélgica, Noruega y Japón estas cifras resultan aun superiores. De igual manera, en economías en transición del centro y este de Europa, como Bulgaria, República Checa, Estonia, Croacia, Polonia o Eslovaquia, las PYME aportan más de 50\% del empleo en el sector formal.( Romero Luna, I 2006)

Con referencia a lo anterior las Pyme desempeñan un papel relevante tanto en las economías desarrolladas como en los países en desarrollo. No obstante, algunos autores han supuesto identificar regularidades en la evolución del tamaño empresarial a lo largo del proceso de desarrollo económico, marcando la predominancia de un tipo de organización u otro en diferentes etapas históricas. (Romero Luna, Isidoro 2006).

Estudioso de la temática como Romero Luna, I (2006) plantea la no existencia de una relación directa y simple entre el nivel de desarrollo y la composición dimensional del tejido empresarial. El tamaño empresarial óptimo en la escala microeconómica es el resultado de un amplio conjunto de factores, entre los cuales están las economías de escala, los costes de transacción o la estructura de los mercados, por lo cual no existe una dimensión óptima de la empresa para cualquier situación y en todo momento. De modo semejante, en la escala macroeconómica, la 
distribución por tamaño del tejido empresarial en una economía determinada es consecuencia de las dotaciones de recursos, la tecnología disponible, el grado de apertura, las instituciones, las pautas de demanda o las tradiciones productivas de cada zona, entre otros condicionantes. Esa estructura dimensional se ve afectada, asimismo, por las transformaciones tecnológicas, económicas y sociopolíticas que continuamente se están produciendo.

Según se ha citado las Pyme se han mostrado capaces de superar las limitaciones impuestas por su tamaño a fin de abordar conductas dinamizadoras, como proyectos de crecimiento, el acceso a mercados de exportación o la introducción de innovaciones, compartiendo con otras Pyme los costes fijos asociados a esas iniciativas. De ese modo, las Pyme, consideradas individualmente, han dejado de ser el objeto de estudio relevante pasando a serlo los sistemas localizados de Pyme vinculadas por relaciones de colaboración (Camisón, 2000), (Romero I. 2006).

Es gratificante expresar que más allá de la infecunda contraposición Pyme -gran empresa, la escucha debe trasladarse hoy día hacia la complementariedad que surge entre ambas. La articulación empresarial, relativa a las vinculaciones verticales Pyme -gran empresa o a las relaciones horizontales entre PYME se manifiesta en la actualidad como componente determinante de la fortaleza y la competitividad del sistema productivo de un territorio. (Romero I. 2003).

\section{Consideraciones finales}

La subsistencia de las pequeñas y medianas empresas (Pymes) está sujeta a superar problemas internos para afrontar los externos y conquistar los resultados financieros proyectados.

Es transcendental que las Pymes se desenvuelvan en un ambiente de mejora continuo, que les permita un crecimiento sostenible en el tiempo, con la finalidad de posicionarse y mantenerse en el mercado, con convenientes fuentes de financiamiento.

En el crecimiento socio-económico de cada país las Pymes componen un factor sustancial, lo que le permite incrementar su desempeño y requerir la implementación de estrategias que beneficien las operaciones, todo con la finalidad de reducir los costos de operación, mejorar la eficiencia de 
los procesos, los niveles de inventario, la calidad de los productos y por supuesto incrementar la productividad.

Las Pymes forjan riqueza y empleo, ya que dinamizan la economía y mejora la gobernabilidad, ya que requieren menores costos de inversión, es el sector que mayormente utiliza insumos y materias primas.

\section{Referencias}

1. América Latina: en busca de pymes más competitivas. (2016). Recuperado en https://www.caf.com/es/actualidad/noticias/2016/06/america-latina-en-busca-de-pymesmas-competitivas/

2. Cervantes Molina, Ximena Paola, \& Oviedo Bayas, Byron. (2018). Las micro, pequeñas, medianas empresas del sector manufacturero: un estudio del contexto de la gestión por procesos en el Cantón El Empalme, Ecuador. Revista Universidad y Sociedad, 10(5), 366371. Recuperado en, http://scielo.sld.cu/scielo.php?script=sci_arttext\&pid=S221836202018000500366\&lng=es\&tlng=es.

3. Camisón, César, (2000) "Reflexiones sobre la Investigación Científica de la PYME", en Investigaciones Europeas de Dirección y Economía de la Empresa, Santiago de Compostela, Academia. Europea de Dirección y Economía de la Empresa, vol. 6, núm. 2, pp. 13-30.

4. Delgado Delgado D D, Chávez Granizo G P. (2018). Las Pymes en el Ecuador y sus fuentes de financiamiento. Recuperado en: https://www.eumed.net/rev/oel/2018/04/pymes-ecuador-financiamiento.html

5. Dini M, Stumpo G. (2018). MIPYMES en América Latina. Recuperado en: https://repositorio.cepal.org/bitstream/handle/11362/44148/1/S1800707_es.pdf

6. Estupiñan, K. (2015). Las Pymes y los sectores de la economía Nacional. Guayaquil: Universidad de Guayaquil. 
7. INEC. (2016). Directorio de Pymes 2016. Obtenido de INEC: Recuperado en : http://www.inec.gob.ec/documentos_varios/presentacion_direc_empresas.pdf

8. Instituto Nacional de Estadística y Geografía (2009), Censo Empresarial 2009. Recuperado en : en www.inegi.org.mx

9. Madrid C. (2018). Administración de la Empresa. Presentación. Gestiopolis Recuperado en: https://www.gestiopolis.com/administracion-la-empresa-presentacion/

10. OECD (2005). SME Entrepreneurship outlook.OECD, Publications: Paris. Recuperado en: https://www.oecd.org/cfe/smes/oecdsmeandentrepreneurshipoutlook-2005edition.htm

11. Perez, W. y G. Stumpo (2002), "La dinámica de las pequeñas y medianas empresas industriales en América Latina y el Caribe”, Pequeñas y medianas empresas industriales en América LatinaCiudad de México, Siglo XXI/Comisión Económica para América Latina y el Caribe (CEPAL),

12. Quintero Reatiga J .2018. Las pymes en Colombia y las barreras para su desarrollo y perdurabilidad. Recuperado

en: https://repository.unimilitar.edu.co/bitstream/handle/10654/17949/QuinteroReatigaJuanSe bastian2018.pdf?sequence $=1 \&$ isAllowed $=\mathrm{y}$

13. Rojas, M., \& Briceño, M. (2007). La Mercadotecnia en las PYMEs manufactureras del sector tradicional y residual del estado Trujillo.Visión Gerencial, 6(2), 316-327. Recuperado http://erevistas.saber.ula.ve/index.php/visiongerencial/article/download/1018/993

14. Romero Luna, Isidoro (2006). Las PYME en la economía global. Hacia una estrategia de fomento empresarial Problemas del Desarrollo. Revista Latinoamericana de Economía, vol. 37, núm. 146, pp. 31-50. Recuperado en https://www.redalyc.org/pdf/118/11820858002.pdf 


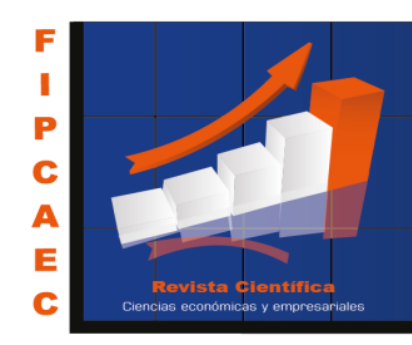

15. Romero, Luna I, (2003). Desarrollo endógeno y articulación productiva. Un análisis del sistema productivo Andaluz, Tesis Doctoral, Sevilla, Universidad de Sevilla,

16. Saavedra G., M L y Hernández C., Y. (2008). Caracterización e importancia de las MIPYMES en Latinoamérica... Actualidad Contable FACES Año 11 No 17,. Mérida. Venezuela. (122-134) Recuperado en https://www.redalyc.org/pdf/257/25711784011.pdf

17. Valdés Díaz de Villegas, J A, \& Sánchez Soto, G A (2012). Las MIPYMES en el contexto mundial: sus particularidades en México. Iberoforum. Revista de Ciencias Sociales de la Universidad Iberoamericana, VII (14), undefined-undefined. ISSN:. Recuperado en : https://www.redalyc.org/articulo.oa?id=2110/211026873005

18. Vergara M S. (2005). Pequeñas y Medianas Empresas en América Latina. Recuperado en:https://www.researchgate.net/publication/228959619_Pequenas_y_Medianas_Empresa s_en_America_Latina

19. Yance Carvajal C, Solís Granda L, Burgos Villamar I, Hermida Hermida L. (2017). Observatorio Economía Latinoamericana. Recuperado en http://www.eumed.net/cursecon/ecolat/ec/2017/pymes-ecuador.html

20. Zapata Guerrero, Edgar Enrique (2004). Las PyMES y su problemática empresarial. Análisis de casos. Revista Escuela de Administración de Negocios, (52),. Recuperado en: https://www.redalyc.org/articulo.oa?id=206/20605209

\section{References}

1. Latin America: in search of more competitive SMEs. (2016). Recovered at https://www.caf.com/es/actualidad/noticias/2016/06/america-latina-en-busca-de-pymesmas-competitivas/

2. Cervantes Molina, Ximena Paola, \& Oviedo Bayas, Byron. (2018). The micro, small, medium enterprises of the manufacturing sector: a study of the context of process 
management in the El Empalme Canton, Ecuador. University and Society Magazine, 10 (5), 366-371.

Retrieved from http://scielo.sld.cu/scielo.php?script=sci_arttext\&pid=S2218$36202018000500366 \& \operatorname{lng}=$ en\&tlng=en.

3. Camisón, César, (2000) "Reflections on the Scientific Research of SMEs", in European Research on Business Administration and Economics, Santiago de Compostela, Academia. European Management and Business Economics, vol. 6, no. 2, pp. 13-30.

4. Delgado Delgado D D, Chávez Granizo G P. (2018). SMEs in Ecuador and their sources of financing. Retrieved from: https://www.eumed.net/rev/oel/2018/04/pymes-ecuadorfinanciamiento.html

5. Dini M, Stumpo G. (2018). MSMEs in Latin America. Retrieved from: https://repositorio.cepal.org/bitstream/handle/11362/44148/1/S1800707_en.pdf

6. Estupiñan, K. (2015). SMEs and sectors of the National economy. Guayaquil: University of Guayaquil.

7. INEC. (2016). SME Directory 2016. Obtained from INEC: Retrieved from: http://www.inec.gob.ec/documentos_varios/presentacion_direc_empresas.pdf

8. National Institute of Statistics and Geography (2009), 2009 Business Census. Retrieved from: www.inegi.org.mx

9. Madrid C. (2018). Businesses Administration. Presentation. Gestiopolis Recovered at: https://www.gestiopolis.com/administracion-la-empresa-presentacion/

10. OECD (2005). SME Entrepreneurship outlook.OECD, Publications: Paris. Retrieved from: https://www.oecd.org/cfe/smes/oecdsmeandentrepreneurshipoutlook2005edition.htm 


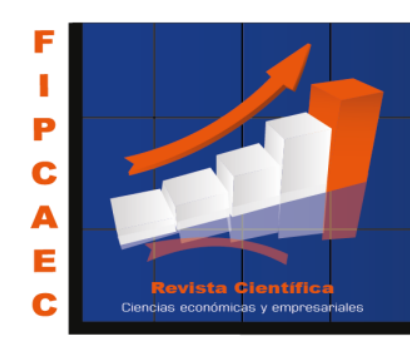

11. Perez, W. and G. Stumpo (2002), "The dynamics of small and medium-sized industrial enterprises in Latin America and the Caribbean," Small and medium-sized industrial enterprises in Latin America, Mexico City, 21st century / Economic Commission for America Latin and the Caribbean (ECLAC),

12. Quintero Reatiga J. 2018. SMEs in Colombia and the barriers to their development and durability. Retrieved from: https://repository.unimilitar.edu.co/bitstream/handle/10654/17949/QuinteroReatigaJuanSe bastian2018.pdf?sequence $=1$ \&isAllowed $=\mathrm{y}$

13. Rojas, M., \& Briceño, M. (2007). Marketing in manufacturing SMEs in the traditional and residual sector of Trujillo state. Management Commission, 6 (2), 316-327. Recovered from http://erevistas.saber.ula.ve/index.php/visiongerencial/article/download/1018/993

14. Romero Luna, Isidoro (2006). SMEs in the global economy. Towards a business development strategy Development Problems. Latin American Journal of Economy, vol. 37, no. 146, pp. 31-50. Retrieved at https://www.redalyc.org/pdf/118/11820858002.pdf

15. Romero, Luna I, (2003). Endogenous development and productive articulation. An analysis of the Andalusian production system, Doctoral Thesis, Seville, University of Seville,

16. Saavedra G., M L and Hernández C., Y. (2008). Characterization and importance of MSMEs in Latin America ... Current Accounting FACES Year $11 \mathrm{~N}^{\mathrm{o}}$ 17,. Merida Venezuela. (122-134) Retrieved at https://www.redalyc.org/pdf/257/25711784011.pdf

17. Valdés Díaz de Villegas, J A, \& Sánchez Soto, G A (2012). MSMEs in the global context: their peculiarities in Mexico. Iberoforum Journal of Social Sciences of the Universidad Iberoamericana, VII (14), undefined-undefined. ISSN:. Retrieved from: https://www.redalyc.org/articulo.oa?id=2110/211026873005 
18. Vergara M S. (2005). Small and Medium Enterprises in Latin America. Retrieved from: https:

//www.researchgate.net/publication/228959619_Pequenas_y_Medianas_Empresas_en_A merica_Latina

19. Yance Carvajal C, Solís Granda L, Burgos Villamar I, Hermida Hermida L. (2017). Latin $\begin{array}{llll}\text { American } & \text { Observatory. } & \text { Retrieved }\end{array}$ http://www.eumed.net/cursecon/ecolat/ec/2017/pymes-ecuador.html

20. Zapata Guerrero, Edgar Enrique (2004). SMEs and their business problems. Case Analysis School of Business Administration Magazine, (52),. Retrieved from: https://www.redalyc.org/articulo.oa?id=206/20605209

(C2018 por el autor. Este artículo es de acceso abierto y distribuido según los términos y condiciones de la licencia Creative Commons Atribución-NoComercial-Compartirlgual 4.0 Internacional (CC BY-NC-SA 4.0) (https://creativecommons.org/licenses/by-nc-sa/4.0/). 